\title{
In-process estimation of time-variant contingently correlated measurands
}

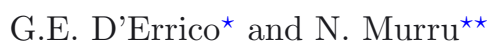 \\ Istituto Nazionale di Ricerca Metrologica (INRIM), Torino, Italy
}

Received: 12 October 2012 / Accepted: 28 October 2012

\begin{abstract}
This paper is devoted to the study and implementation of real-time techniques for the estimation of time-varying, contingently correlated quantities, and relevant uncertainty. An estimation algorithm based on a metrological customization of the Kalman filtering technique is presented, starting from a Bayesian approach. Moreover, a fuzzy-logic routine for real-time treatment of possible outliers is incorporated in the overall software procedure. The system applicability is demonstrated by results of simulations performed on dimensional measurement models.
\end{abstract}

Keywords: Kalman filter; time-varying measurands; real-time estimation; correlation; fuzzy-logic based outlier treatment

\section{Introduction}

In the context of in-process metrology, accurate statistical analyses are important to optimize real-time estimation of measurands and related uncertainties. The, Kalman filtering (KF) technique [1] is optimal under diverse criteria [2]. Moreover, it is widely used long since and it is successfully being applied in several fields (see, e.g., [2-5]).

In [6] and [7] a novel application of KF was developed in the field of dimensional metrology. In [6], such a customization is applied to coordinate measuring machines (CMMs). In [7], the measurands are vectorial quantities that can vary during time, according to some specified patterns. Some simulations are executed in order to discuss the algorithm performance. Both papers consider the measurands as unknown parameters, modelled in term of mutually independent normal random variables (RVs). In the present paper, the model is improved by taking into account possible correlations among RVs, so to manage dependence among measurands.

The problem is approached using the covariance matrix, which is an established technique in the KF (see, e.g., [8-10]). Finally, a routine is proposed to perform an outlier treatment based on fuzzy logics (applicability of fuzzy logics in uncertainty treatment is dealt with in [11]).

Even if the KF is robust by design (against, e.g., initial uncertainty and round-off errors) its performance could be affected by occurrence of possible outliers [12]. In [13] a strategy, based on a fuzzy-logic approach, was proposed for possible outlier treatment. In the present paper, such a strategy is embedded in the estimation procedure.

\footnotetext{
^ Correspondence: g.derrico@inrim.it

${ }^{\star \star}$ Supported by an INRIM's post-doc fellowship
}

The paper is organized as follows. Section 2 is devoted to the algorithm formulation. A metrological customization of the $\mathrm{KF}$ is derived starting from the Bayes theorem by using Gaussian multivariate distribution functions (MDFs) and managing correlations (if any) via Gaussian copula (Sect. 2.1). The fuzzy outlier treatment presented in [13] is briefly recalled and embedded in the KF estimation algorithm (Sect. 2.2).

Section 3 presents the overall software (SW) architecture by means of a Simulink ${ }^{\mathrm{TM}}$ diagram. ${ }^{1}$ In Section 4 , some application examples are shown, where the estimation targets are two rectangular surfaces with a common edge. Section 5 contains some concluding remarks.

\section{Algorithm formulation}

\subsection{Metrological customization of KF technique}

The standard $\mathrm{KF}$ is a recursive technique to estimate the state vector $\boldsymbol{x}_{k}=\left(\boldsymbol{x}_{k}(1), \ldots \boldsymbol{x}_{k}(i) \ldots, \boldsymbol{x}_{k}(m)\right)(i=$ $1, \ldots, m$, where $m$ is the vector dimension, and $0 \leqslant k \leqslant L$ the discrete time) of a linear process described by the equation:

$$
\boldsymbol{x}_{k+1}=\boldsymbol{A}_{k} \boldsymbol{x}_{k}+\boldsymbol{B}_{k} \boldsymbol{u}_{k}+\boldsymbol{\eta}_{k}
$$

where $\boldsymbol{x}_{k}, \boldsymbol{u}_{k}$ (optional control input), and $\boldsymbol{\eta}_{k}$ (white noise) are vectors, and $\boldsymbol{A}_{k}, \boldsymbol{B}_{k}$ are matrices which relate the process state at the step $k+1$ with the $k$ th process

\footnotetext{
1 Identification of commercial products in this paper does not imply recommendation or endorsement, nor does it imply that the products identified are necessarily the best available for the purpose.
} 
state and with the $k$ th control input, respectively. The (indirect) measurement $\boldsymbol{z}_{k}$ of $\boldsymbol{x}_{k}$ is modeled as follows:

$$
\boldsymbol{z}_{k}=\boldsymbol{H}_{k} \boldsymbol{x}_{k}+\boldsymbol{v}_{k}
$$

where the vector $\boldsymbol{v}_{k}$ is introduced due to the measurement uncertainty and $\boldsymbol{H}_{k}$ relates the (observable) output vector $\boldsymbol{z}_{k}$ with the (internal) state $\boldsymbol{x}_{k}$. In metrology terms, $\boldsymbol{z}_{k}$ and $\boldsymbol{x}_{k}$ represent the measured quantity values and the theoretical measurand, respectively. The vector $\boldsymbol{u}_{k}$ is used to track the time-evolution of the theoretical pattern of $\boldsymbol{x}_{k+1}$. In these terms, the model is translated into the context of measurement science. The estimation is provided balancing the measured quantity $\boldsymbol{z}_{k}$ with an a-priori estimation vector $\boldsymbol{x}_{k}^{-}$by using the Kalman gain matrix $\boldsymbol{K}_{k}$ :

$$
\boldsymbol{y}_{k}=\boldsymbol{x}_{k}^{-}+\boldsymbol{K}_{k}\left(\boldsymbol{z}_{k}-\boldsymbol{H}_{k} \boldsymbol{x}_{k}^{-}\right), \quad 0 \leqslant k \leqslant L
$$

where $\boldsymbol{y}_{\mathbf{k}}$ is the estimation of $\boldsymbol{x}_{\mathbf{k}}$ provided by the KF and

$$
\begin{aligned}
& \boldsymbol{x}_{0}^{-}=\boldsymbol{y}_{-1} \\
& \boldsymbol{x}_{k}^{-}=A_{k-1} \boldsymbol{y}_{k-1}+\boldsymbol{B}_{k-1} \boldsymbol{u}_{k-1}, 1 \leqslant k \leqslant L
\end{aligned}
$$

where $\boldsymbol{y}_{-1}$ is an a-priori expert judgment of the measurand vector at the initial state. The gain matrix $\boldsymbol{K}_{k}$ is constructed using the covariance matrix of the RVs relevant to the components of the vector $\boldsymbol{x}_{k} . \boldsymbol{K}_{k}$ is obtained by minimizing the mean-square-error $E\left[\left(\boldsymbol{y}_{k}-\boldsymbol{x}_{k}\right)\left(\boldsymbol{y}_{k}-\boldsymbol{x}_{k}\right)^{\boldsymbol{T}}\right]$ where $E[\cdot]$ stands for expectation and superscript $T$ for transposition.

In [6], the KF technique has been customized for metrology usage, dealing with scalar time-invariant quantities. In [7], such an approach has been generalized to time-varying measurand vectors, whose components were supposed mutually independent.

In the present paragraph, the approach is further developed, so to take into account possible correlations among the measurand vector components; moreover, an outlier treatment incorporated in the estimation procedure is developed in Section 2.2.

Let $X$ and $Z$ represent the stochastic counterparts of $\boldsymbol{x}_{k-}$ and $\boldsymbol{z}_{k}$, respectively. The Kalman gain matrix $\boldsymbol{K}_{k}$ can be derived by using the Bayes theorem:

$$
f(X \mid Z)=f(Z \mid X) f(X)\left[\int_{\Delta} f(Z \mid X) f(X) \mathrm{d} X\right]^{-1}
$$

where $f$ is a probability density function (PDF), $f(X \mid Z)$ is the posterior density, $f(X)$ is the prior density, $f(Z \mid X)$ is the likelihood, and the integration (over the domain of definition $\Delta$ of $X$ ) gives rise to a normalization factor (the denominator).

The following treatment will be based on the hypothesis of Gaussian RVs to model the vector measurands. In order to manage possible correlations, the Gaussian copula is a useful tool to obtain Gaussian MDFs from any vector of univariate cumulative distribution functions (CDFs): a copula is a function that couples univariate (marginal) cumulative distributions into a joint MDF, whose expression includes original correlations among marginal univariates [14].

Let $N(\boldsymbol{\mu}, \boldsymbol{\Sigma})$ denote a Gaussian MDF, where $\boldsymbol{\mu}$ is the vector of mean values and $\Sigma$ is the covariance matrix. A Gaussian copula $C$ is a particular family of copulas such that, given $n$ marginals $h_{1}, \ldots, h_{n}, C\left(h_{1}, \ldots, h_{n}\right)=$ $G_{\Sigma}\left(g_{-1}\left(h_{1}\right), \ldots, g_{-1}\left(h_{n}\right)\right)=N(\boldsymbol{\mu}, \boldsymbol{\Sigma})$, where $G_{\Sigma}$ is the $n$-variate Gaussian CDF with covariance matrix $\boldsymbol{\Sigma}$ and $g$ is the univariate standard Gaussian.

$$
\begin{aligned}
& \text { Let } f(X)=N\left(\boldsymbol{x}_{k}^{-}, \boldsymbol{P}_{k-1}\right), f(Z \mid X)=N\left(\boldsymbol{z}_{k}, \boldsymbol{R}\right) \text { and } \\
& \boldsymbol{P}_{-1}=\boldsymbol{\Pi}_{-1}, \boldsymbol{P}_{k}=\left(\boldsymbol{P}_{k-1}^{-1}+\boldsymbol{R}^{-1}\right)^{-1}, 1 \leqslant k \leqslant L
\end{aligned}
$$

with $\boldsymbol{\Pi}_{-1}$ and $\boldsymbol{R}$ symmetric covariance matrices initialized according to prior knowledge (based on an expert judgment): diagonal entries can be used for type B uncertainty treatment (see guide [15]) and other non-zero entries represent mutual correlation coefficients. Equation (5) states that $f(X \mid Z)$ is proportional to $N\left(\boldsymbol{y}_{k}\right.$, $\left.\boldsymbol{P}_{k}\right)=N\left(\boldsymbol{x}_{k}^{-}, \boldsymbol{P}_{k-1}\right) N\left(\boldsymbol{z}_{k}, R\right)$, where

$\boldsymbol{y}_{k}=\left(\boldsymbol{P}_{k-1}^{-1}+\boldsymbol{R}^{-1}\right)^{-1}\left(\boldsymbol{P}_{k-1}^{-1} \boldsymbol{x}_{k}^{-}+\boldsymbol{R}^{-1} \boldsymbol{z}_{k}\right), 0 \leqslant k \leqslant L$.

The final estimates are provided in terms of $E(f(X \mid Z))$ together with standard uncertainty (after square roots of diagonal entries from the covariance matrix) evaluated at $k=L$ (see $[7,16])$. Equations (4)-(7) form the recursive algorithm used in this paper for KF metrological customization.

\subsection{Fuzzy logic-based modeling of outlier detection and treatment}

The algorithm is enriched by a routine for real-time treatment of possible outliers that can affect the estimation results. Several statistical tests have been proposed to manage this problem, such as Dixon's test and Grubbs' one: a standard also deals with such a problem [17].

However, tests of orthodox statistics kind - besides being prone to Bayesian criticism - are also subject to statistical hypotheses, mainly randomness and independence of observations [18] that impose applicability limitation in order to preserve consistency.

In [13] a fuzzy approach is proposed aiming at coping with this situation, by modeling the problem of outliers in terms of fuzzy sets, so to treat the processed observations by means of purposely defined "outlierness" degrees.

The fuzzy strategy, based on a 2-input/1-output inference scheme [13], operates component-wise on involved vectors, by use of the following scalar quantities: $z$ a measurand observation, $\eta$ an a-priori estimation of the measurand, $d(z, \eta)=|z-\eta|$ their relative distance, and $\sigma$ the a-priori estimation uncertainty.

In the inference scheme (Mamdani model $[19,20]$ ), one input is the fuzzyfication of the distance $d(z, \eta)$ and the other input is the fuzzyfication of the percentage uncertainty $\sigma \%=100 \sigma / \eta$, both obtained by properly defined fuzzy sets and related membership functions 


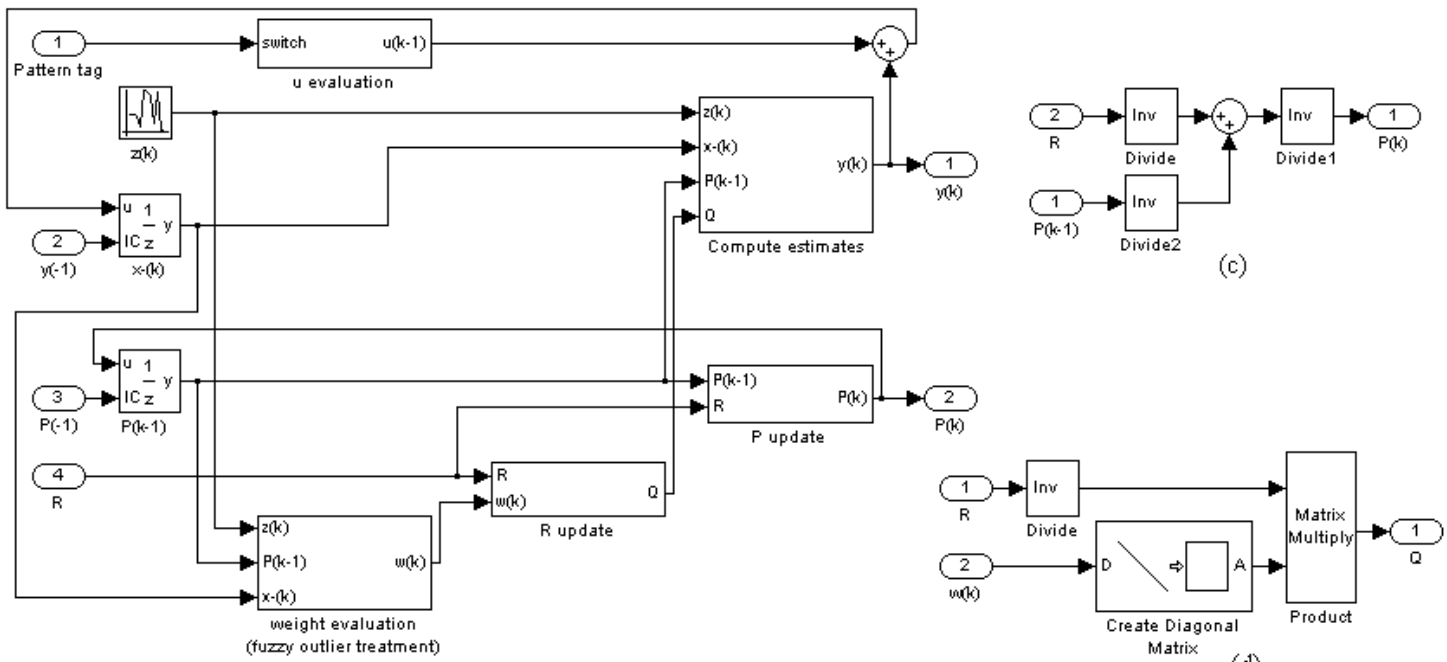

(a)

(d)

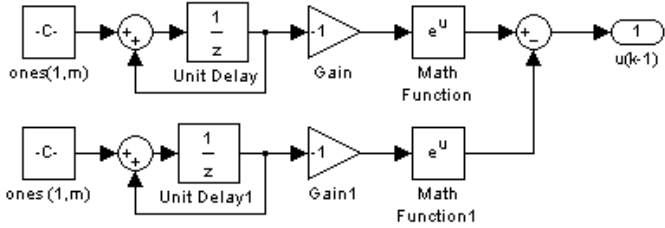

(b)

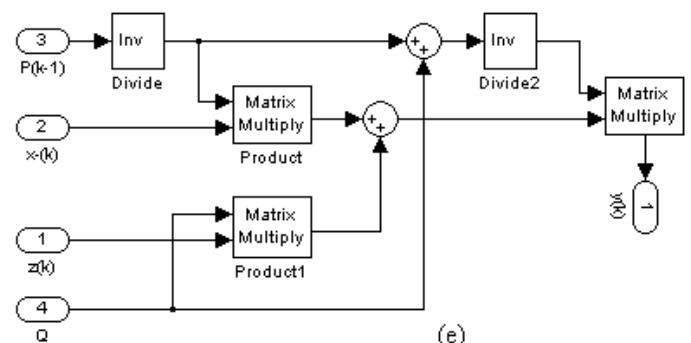

(e)

Fig. 1. Simulink ${ }^{\mathrm{TM}}$ diagram (a) and blocks: (b) "u evaluation"; (c) "P update"; (d) "R update"; (e) "compute estimates".

(see [13] for details). The output is the outlierness degree $0 \leqslant \rho(z) \leqslant 1$ relative to the possible outlying observation $z$, which is obtained by application of the centroid defuzzification method (ten composition rules are used after [13]). The fuzzy treatment is activated if the condition $2 \sigma<d(z, \eta)<5 \sigma$ is satisfied, otherwise: if $d(z, \eta) \leqslant$ $2 \sigma, z$ is defined a "full inlier" (thus $\rho(z)$. = 0 ); else, if $d(z, \eta) \geqslant 5 \sigma, z$ is defined a "full outlier" (so that $\rho(z)=1)$. After this, for estimation purpose, the outlierness degree is conveniently translated into an outlierness weight $w(z)=1-\rho(z)$.

In the present paper - moving from mono-dimensional (the case-study in [13]) to multi-dimensional measurands this kind of weight is used for estimation of time-varying vector quantities after integration in the $\mathrm{KF}$ routine. In the $\mathrm{KF}$ routine described in the previous subsection, at the step $k$, the vector $\boldsymbol{z}_{k}$ is the measurand observation, $\boldsymbol{x}_{k}^{-}$is the a-priori measurand estimation, and $\boldsymbol{P}_{k-1}$ is the covariance matrix elaborated to deduce the uncertainty related to $\boldsymbol{x}_{k}^{-}$.

To apply the outlier fuzzy treatment to vectorial quantities, a component wise treatment can be performed. For every $i=1, \ldots, m$, let $\boldsymbol{z}_{k}(i)$ and $\boldsymbol{x}_{k}^{-}(i)$ be the $i$ th component of $\boldsymbol{z}_{k}$ and $\boldsymbol{x}_{k}^{-}$respectively, and let $P_{k-1}(i, i)$ be the $i$ th diagonal entry of the matrix $\boldsymbol{P}_{k-1}$. The outlier fuzzy treatment is embedded in the KF by use of $z=$ $\boldsymbol{z}_{k}(i), \eta=\boldsymbol{x}_{k}(i), \sigma^{2}=P_{k-1}(i, i)$. For the measurement vector $\boldsymbol{z}_{k}$, an outlierness weight $w_{k}(i)$ is associated to the measurement $\boldsymbol{z}_{k}(i)$, giving rise to the outlierness weight vector $\boldsymbol{w}_{k}=\left(w_{k}(1), \ldots w_{k}(i) \ldots, w_{k}(m)\right)$.

After evaluation, the weight $\boldsymbol{w}_{k}$ must be incorporated in the KF routine. Equation (7) that provides the estimation $\boldsymbol{y}_{k}$ in terms of a weighted mean of $\boldsymbol{x}_{k}^{-}$ and $\boldsymbol{z}_{k}$ can be rewritten $\boldsymbol{y}_{k}=\left(\boldsymbol{P}_{k-1}^{-1} \boldsymbol{x}_{k}^{-}+\boldsymbol{R}^{-1} \boldsymbol{z}_{k}\right)\left(\boldsymbol{P}_{k-1}^{-1}+\right.$ $\left.\boldsymbol{R}^{-1}\right)^{-1}$, making clear that $\boldsymbol{R}^{-1}$ is the weight matrix of $\boldsymbol{z}_{k}\left(\boldsymbol{R}\right.$ and its inverse $\boldsymbol{R}^{-1}$ are diagonal matrices, i.e., mutual independence of measurement vector components is assumed).

For fuzzy treatment purpose, $\boldsymbol{R}^{-1}$ must be scaled in terms of a diagonal matrix $\boldsymbol{Q}$, to take into account $\boldsymbol{w}_{k}$ as follows:

$$
Q(i, i)=R^{-1}(i, i) w_{k}(i), 1 \leqslant i \leqslant m
$$

Therefore, the measurand estimation in the KF is given by

$$
\boldsymbol{y}_{k}=\left(\boldsymbol{P}_{k-1}^{-1}+\boldsymbol{Q}\right)^{-1}\left(\boldsymbol{P}_{k-1}^{-1} \boldsymbol{x}_{k}^{-}+\boldsymbol{Q} \boldsymbol{z}_{k}\right), \quad 0 \leqslant k \leqslant L .
$$

\section{Software architecture}

The algorithm developed in Section 2 has been implemented to simulate real-time estimation of multidimensional time-varying measurands. The realized SW architecture is illustrated in Figure 1 by means of a Simulink ${ }^{\mathrm{TM}}$ diagram. In the implemented SW procedure, 


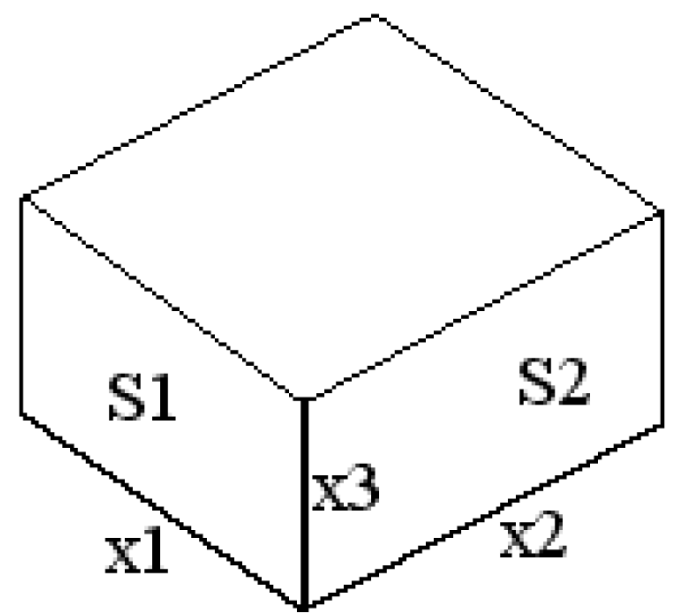

Fig. 2. Rectangular surfaces $S_{1}$ and $S_{2}$ (measurands).

the measurands are time-varying quantities, which are supposed to evolve according to patterns specified through the input "Pattern tag" in the diagram.

The possible patterns so far available are linear, sawtooth, triangular wave, square wave, and sine wave, exponential and parabolic shapes [7]. The inputs $\boldsymbol{y}_{-1}, \boldsymbol{P}_{-1}$, and $\boldsymbol{R}$ must be pre-set by an expert operator to initialize the routine.

At each step $k$ the routine operates as depicted in Figure 1a. The routine is fed by a measurement $\boldsymbol{z}_{k}$. The vector $\boldsymbol{x}_{k}^{-}$is evaluated putting in equation (4) $\boldsymbol{A}_{k-1}=$ $\boldsymbol{B}_{k-1}=\boldsymbol{I}$ (I identity matrix $) ; \boldsymbol{u}_{k-1}$ is built in the "u evaluation" block according to the selected pattern: in Figure $1 \mathrm{~b}$, an example (for the exponential shape) is shown. In the "P update" block (Fig. 1c), the matrix $\boldsymbol{P}_{k-1}$ is evaluated according to equation $(6) ; \boldsymbol{P}_{k}$ is then used to compute the standard deviations, square roots of $P_{k-1}(i, i)$. The matrix $\boldsymbol{R}$ is transformed into $\boldsymbol{Q}$ ("R update" block in Fig. 1d), see equation (8); $\boldsymbol{w}_{k}$ is evaluated in the block "weight evaluation (fuzzy outlier treatment)" (see Sect. 2.2). Finally, equation (9) is implemented in the "compute estimates" (Fig. 1e) block whose output provides the measurand estimation $\boldsymbol{y}_{k}$.

\section{Simulation: a case-study}

The algorithm behavior is presented and discussed with application to some simulations performed in MATLAB $^{\text {TM }}$. The SW system performance is tested on a case-study where measurands are the areas of two rectangular surfaces $S_{1}$ and $S_{2}$ with a common edge $x_{3}$ (Fig. 2): use of $x_{3}$ to calculate both areas introduces correlations between the components of the measurand vector $\left(S_{1}, S_{2}\right)$. Since $S_{1}=S_{2} x_{1} / x_{2}$, a linear correlation (Pearson coefficient) can properly describe such a model. However, taking into account randomness, the routine is able to process also different correlations (Spearman and Kendall coefficients), which can be entered in the non-diagonal entries of $\boldsymbol{P}_{-1}$ by an expert operator.
Table 1. Measured $(\boldsymbol{z})$, theoretical $(\boldsymbol{x})$, and estimated $(\boldsymbol{y})$ vectors of Figure 3.

\begin{tabular}{cccc}
\hline \multicolumn{4}{c}{$\boldsymbol{y}_{-}=(2.97,6.21)$} \\
$k$ & $\boldsymbol{z}_{k}$ & $\boldsymbol{x}_{k}$ & $\boldsymbol{y}_{k}$ \\
\hline 0 & $(2.70,4.42)$ & $(2.99,5.81)$ & $(2.66,5.43)$ \\
1 & $(4.50,4.80)$ & $(5.02,5.81)$ & $(4.69,5.22)$ \\
2 & $(5.16,5.43)$ & $(5.18,5.81)$ & $(4.94,5.28)$ \\
3 & $(3.57,6.74)$ & $(3.33,5.81)$ & $(3.11,5.56)$ \\
4 & $(1.46,6.92)$ & $(1.17,5.81)$ & $(0.92,5.78)$ \\
5 & $(1.24,4.83)$ & $(0.69,4.87)$ & $(0.50,4.62)$ \\
6 & $(2.76,4.57)$ & $(2.32,4.87)$ & $(2.31,4.62)$ \\
7 & $(4.88,5.60)$ & $(4.57,4.87)$ & $(4.74,4.72)$ \\
8 & $(5.43,4.92)$ & $(5.37,4.87)$ & $(5.58,4.74)$ \\
9 & $(4.14,5.36)$ & $(3.98,4.87)$ & $(4.10,4.79)$ \\
\hline
\end{tabular}

Table 2. Measured $(\boldsymbol{z})$, theoretical $(\boldsymbol{x})$, and estimated $(\boldsymbol{y})$ vectors of Figure 4.

\begin{tabular}{cccc}
\hline \multicolumn{4}{c}{$\boldsymbol{y}_{-1}=(1.35,4.56)$} \\
$k$ & $\boldsymbol{z}_{k}$ & $\boldsymbol{x}_{k}$ & $\boldsymbol{y}_{k}$ \\
\hline 0 & $(4.83,3.51)$ & $(2.49,3.09)$ & $(3.62,4.05)$ \\
1 & $(1.30,1.36)$ & $(2.45,3.10)$ & $(2.55,2.87)$ \\
2 & $(1.02,3.92)$ & $(2.41,3.12)$ & $(2.10,3.18)$ \\
3 & $(1.02,2.40)$ & $(2.37,3.15)$ & $(1.81,3.02)$ \\
4 & $(1.46,3.49)$ & $(2.33,3.19)$ & $(1.71,3.15)$ \\
5 & $(3.50,2.38)$ & $(2.29,3.25)$ & $(1.94,3.10)$ \\
6 & $(1.46,1.63)$ & $(2.25,3.32)$ & $(1.82,2.97)$ \\
7 & $(1.17,3.43)$ & $(2.21,3.40)$ & $(1.70,3.12)$ \\
8 & $(2.44,3.04)$ & $(2.17,3.50)$ & $(1.74,3.22)$ \\
9 & $(4.18,2.51)$ & $(2.13,3.60)$ & $(1.92,3.28)$ \\
\hline
\end{tabular}

Measurements of $x_{1}, x_{2}, x_{3}$ are modeled by independent RVs and the measurement vector $\boldsymbol{z}_{k}$ is (indirectly) obtained by $S_{1}=x_{1} x_{3}$ and $S_{2}=x_{2} x_{3}$. While $x_{3}$ is supposed a non-varying quantity for the seek of simplicity, $x_{1}$ and $x_{2}$ are supposed time-varying quantities due to, e.g., temperature fluctuations: $S_{1}$ and $S_{2}$ follow the same patterns of $x_{1}$ and $x_{2}$, respectively.

Figures 3 and 4 (whose simulation data are contained in Tables 1 and 2, respectively) show the algorithm behavior without outlier treatment. Figures $3 a$ and 4 a represent the first component of the measurand vector (surface $S_{1}$ ) time-varying with sine and linear pattern, respectively. Figures $3 \mathrm{~b}$ and $4 \mathrm{~b}$ represent the second component (surface $S_{2}$ ), which follows a square wave and an exponential shape pattern, respectively.

For simulation purpose, measurements of $x_{1}, x_{2}$, and $x_{3}$ are obtained at each step by random generators, as follows: in Figure $3, x_{1}, x_{2}$, and $x_{3}$ are sampled from normal marginal distributions and Pearson coefficient has been used; in Figure 4 (with Kendall coefficient), $x_{1}$ and $x_{2}$ are obtained from uniform marginal distributions, for $x_{3}$ a gamma marginal distribution has been used. The entries of $2 \times 2$ matrices $\boldsymbol{P}_{-1}$ and $\boldsymbol{R}$ are: as regards Figure 3: $P_{-1}(1,1)=P_{-1}(2,2)=0.40$, 

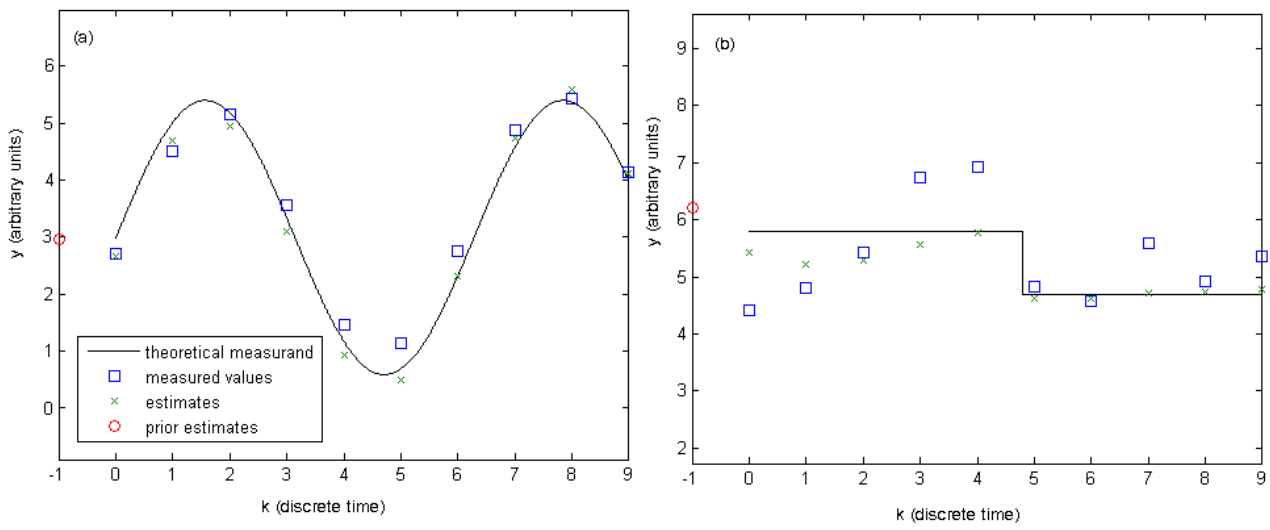

Fig. 3. Cyclic patterns for $S_{1}(\mathrm{a})$ and $S_{2}(\mathrm{~b})$ : simulation results.

Table 3. Measured $(\boldsymbol{z})$, theoretical $(\boldsymbol{x})$, and weight $(\boldsymbol{w})$ vectors of Figure 5; Estimated vectors $(\boldsymbol{y})$ of Figures 5a and 5b; Estimated vectors $(\boldsymbol{y} *)$ of Figures $5 \mathrm{c}$ and $5 \mathrm{~d}$.

\begin{tabular}{ccccccc}
\hline \multicolumn{5}{c}{$\boldsymbol{y}_{-1}=(4.33,6.95)$} \\
$k$ & $\boldsymbol{z}_{k}$ & $\boldsymbol{x}_{k}$ & $\boldsymbol{y}_{k}$ & $\boldsymbol{y}_{k}$ & $\boldsymbol{w}_{k}$ \\
\hline 0 & $(3.61,6.28)$ & $(4.23,6.60)$ & $(4.39,6.84)$ & $(4.39,6.84)$ & $(1.00,1.00)$ \\
1 & $(2.72,5.46)$ & $(4.22,6.41)$ & $(3.84,6.45)$ & $(4.12,6.55)$ & $(0.50,0.50)$ \\
2 & $(8.37,6.90)$ & $(4.21,6.22)$ & $(4.66,6.32)$ & $(4.18,6.44)$ & $(0.00,1.00)$ \\
3 & $(3.34,7.83)$ & $(4.20,6.13)$ & $(4.32,6.37)$ & $(3.40,6.44)$ & $(1.00,0.50)$ \\
4 & $(4.10,4.66)$ & $(4.19,6.32)$ & $(4.31,6.45)$ & $(4.02,6.60)$ & $(1.00,0.22)$ \\
5 & $(6.63,0.05)$ & $(4.18,6.51)$ & $(4.56,6.25)$ & $(4.11,6.74)$ & $(0.20,0.00)$ \\
6 & $(2.70,1.16)$ & $(4.17,6.50)$ & $(4.32,5.94)$ & $(4.01,6.70)$ & $(0.50,0.00)$ \\
7 & $(2.37,5.74)$ & $(4.16,6.31)$ & $(4.14,5.74)$ & $(3.90,6.47)$ & $(0.50,1.00)$ \\
8 & $(1.06,3.82)$ & $(4.14,6.12)$ & $(3.84,5.42)$ & $(3.90,6.25)$ & $(0.00,0.00)$ \\
9 & $(6.33,4.35)$ & $(4.13,6.23)$ & $(4.11,5.50)$ & $(3.91,6.32)$ & $(0.22,0.22)$ \\
\hline
\end{tabular}

$P_{-1}(1,2)=P_{-1}(2,1)=0.43 ; R(1,1)=R(2,2)=0.5$ $R(1,2)=R(2,1)=0$; as regards Figure $4: P_{-1}(1,1)=$ $0.84, P_{-1}(2,2)=0.75, P_{-1}(1,2)=P_{-1}(2,1)=0.39$; $R(1,1)=R(2,2)=0.35, R(1,2)=R(2,1)=0$.

Uncertainties relative to prior estimate and measurements are close to each other in the case of Figure 3, while in Figure 4, measurements uncertainty is less than that of prior estimate. Activation of the fuzzy outlier treatment is recommended when measurement uncertainty is significantly greater than prior estimate uncertainty: for this reason it is not activated in the simulations reported in Figures 3 and 4.

In these simulations, the algorithm is convergent and efficient, so that most estimated values are closer than measured ones and prior knowledge to the theoretical measurand pattern.

In Figure 5 (see Tab. 3 for data), measurements uncertainty is as large as required to activate the fuzzy outlier treatment in the KF routine. The criterion for outlier detection is based on matching $\boldsymbol{z}_{k}$ against $\boldsymbol{x}_{k}^{-}$: thus a majority of outlying values may result during a simulation, as in Figures $5 \mathrm{c}$ and $5 \mathrm{~d}$. Measurements are obtained by use of normal random functions and the Spearman coefficient describes correlations between $S_{1}$ and $S_{2}$; the entries of $2 \times 2$ matrices $\boldsymbol{P}_{-1}$ and $\boldsymbol{R}$ are: $P_{-1}(1,1)=30$,
$P_{-1}(2,2)=0.20, P_{-1}(1,2)=P_{-1}(2,1)=0.94 ; R(1,1)=$ $0.9, R(2,2)=1, R(1,2)=R(2,1)=0$.

A comparison between the algorithm performance with and without outlier treatment is shown in the panels of Figure 5. Figure 5a (surface $S_{1}$, linear pattern) and Figure 5b (surface $S_{2}$, triangular wave) display the algorithm trend when the treatment is off. In Figure 5c (surface $S_{1}$, linear pattern) and Figure $5 \mathrm{~d}$ (surface $S_{2}$, triangular wave), the treatment is on. Comparing Figures 5a and $5 \mathrm{c}$, it can be noted that at $k=1, k=2, k=5$, and $k=8$ the effect of outlierness weights is to maintain the estimates in Figure 5c closer to the theoretical measurand. Similarly, by contrasting Figures 5b and 5d at $k=8$ and $k=9$, a better performance can be noted in Figure 5d.

\section{Conclusion}

An integrated software system for real-time estimation and candidate outlier treatment has been developed with application to time-varying multi-dimensional measurands.

- The estimation strategy implements a metrological customization of the KF technique, taking into account 

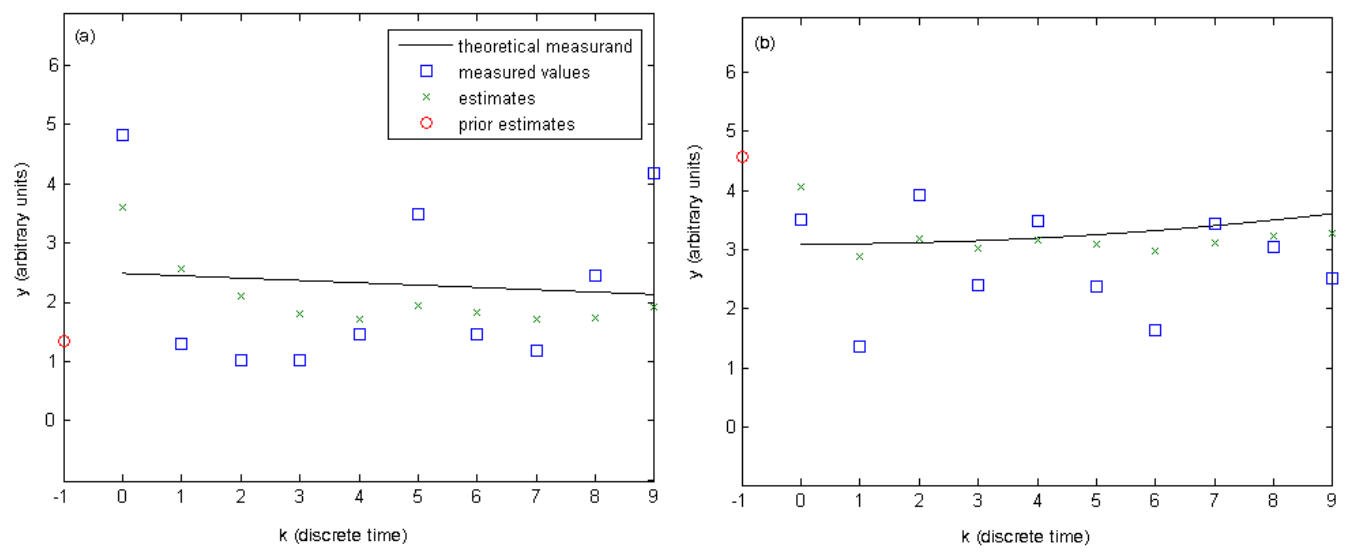

Fig. 4. Acyclic patterns for (a) $S_{1}$ and (b) $S_{2}$ : simulation results.
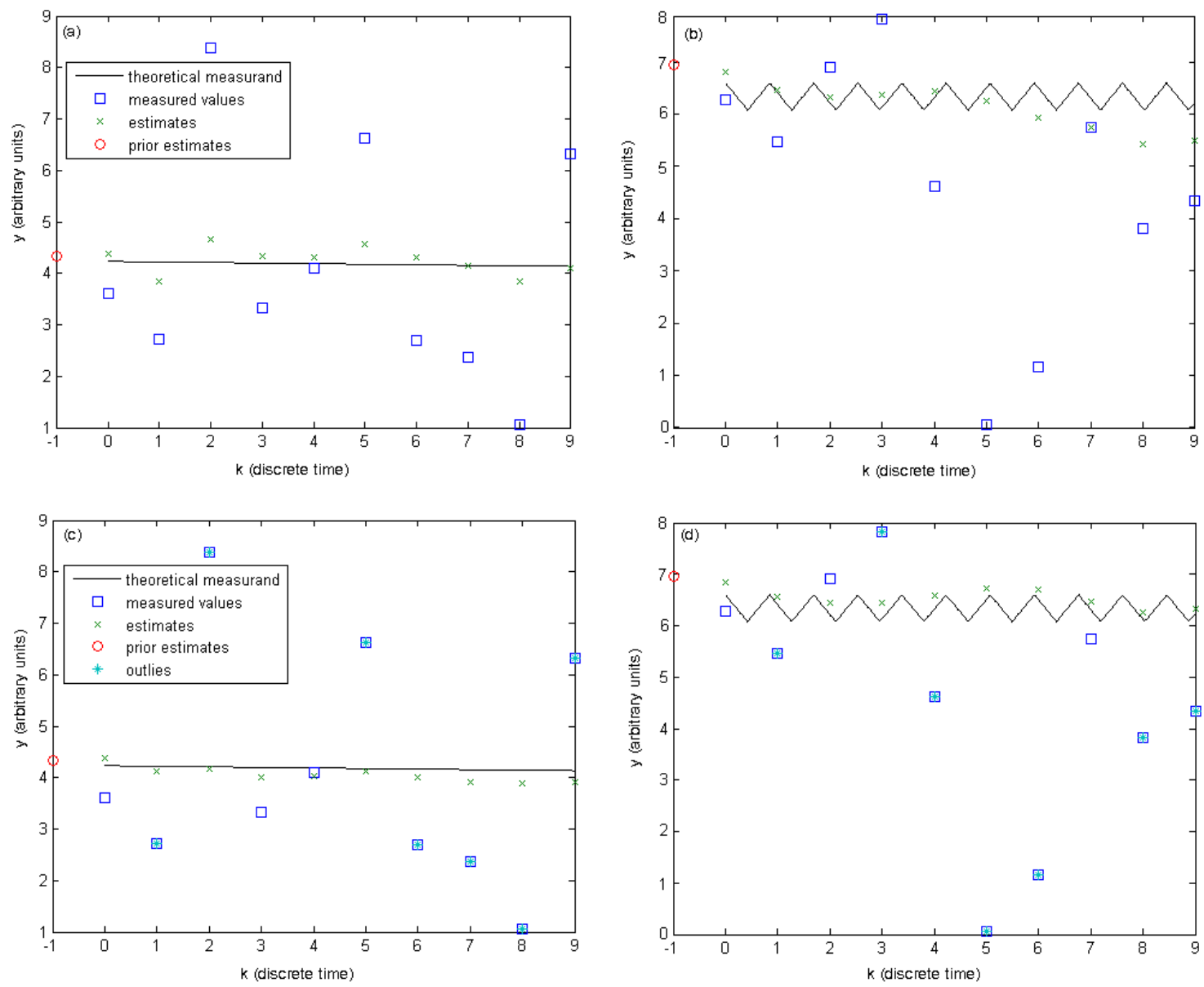

Fig. 5. Comparison between $\mathrm{KF}$ routine with fuzzy outlier treatment off (a, b) or on (c, d).

possible statistical correlation of measurands and related uncertainty evaluation.

- Occurrence of suspected outliers in dynamic measurements is modeled in fuzzy-logic terms for real-time detection and processing.

- The overall SW performance is tested by means of simulation results based on dimensional measurement data: the system's efficiency and convergence are demonstrated.

\section{References}

1. R.E. Kalman, A new approach to linear filtering and prediction problems, Trans. ASME D, J. Basic Eng. 82, 35-45 (1960)

2. H.W. Sorenson, Least-squares estimation: from Gauss to Kalman, IEEE Spectrum 7, 63-68 (1970)

3. C. Mitsantisuk, S. Katsura, K. Ohishi, Kalman-filter-based sensor integration of variable power assist control based on 
human stiffness estimation, IEEE Trans. Ind. Electron. 56 3897-3905 (2009)

4. N. Salvatore, A. Caponio, F. Neri, S. Stasi, G.L. Cascella, Optimization of delayed-state Kalman-filter-based algorithm via differential evolution for sensorless control of induction motors, IEEE Trans. Ind. Electron. 57, 385-394 (2010)

5. W.L. Chan, C.S. Lee, F.B. Hsiao, Real-time approaches to the estimation of local wind velocity for a fixed-wing unmanned air vehicle, Meas. Sci. Technol. 22, 105203 (2011)

6. G.E. D'Errico, À la Kalman filtering for metrology tool with application to coordinate measuring machines, IEEE Trans. Ind. Electron. 59, 4377-4382 (2012)

7. G.E. D'Errico, N. Murru, An algorithm for concurrent estimation of time-variant quantities, Meas. Sci. Technol. 23, 045008 (2012)

8. R.K. Mehra, On the identification of variances and adaptive Kalman filtering, IEEE Trans. Autom. Control AC15, 175-184 (1970)

9. K.A. Myers, B.D. Tapley, Adaptive sequential estimation with unknown noise statistics, IEEE Trans. Autom. Control 21, 520-523 (1976)

10. B.J. Odelson, M.R. Rajamani, J.B. Rawlings, A new autocovariance least-squares method for estimating noise covariances, Automatica 42, 303-308 (2006)

11. G.E. D'Errico, Paradigms for uncertainty treatments: a comparative analysis with application to measurement, Measurement 42, 494-500 (2009)
12. Z.M. Durovic, B.D. Kovacevic, Robust estimation with unknown noise statistics, IEEE Trans. Autom. Control 44, 1292-1296 (1999)

13. G.E. D'Errico, N. Murru, Fuzzy treatment of candidate outliers in measurements, Advances in Fuzzy Systems 2012, Article ID 783843 (2012)

14. R.B. Nelsen, An introduction to Copulas, 2nd edn. (Springer, New York, 2007)

15. BIPM, IEC, IFCC, ISO, IUPAC, IUPAP, and OIML, Evaluation of measurement data-Guide to the expression of uncertainty in measurement (GUM 1995 with minor corrections), JCGM 100 (2008)

16. BIPM, IEC, IFCC, ISO, IUPAC, IUPAP, and OIML, Evaluation of measurement data-Supplement 1 to the "Guide to the expression of uncertainty in measurement"Propagation of distributions using a Monte Carlo method, JCGM 101 (2008)

17. ASTM E178-08, Standard practice for dealing with outlying observations, American Society for Testing and Materials (2008)

18. G.E. D'Errico, Testing for outliers based on Bayes rule, in Proc. XIX IMEKO World Congress on Fundamental and Applied Metrology, Lisbon, Portugal, 2009

19. L.A. Zadeh, Fuzzy sets, Inf. Control 8, 338-353 (1965)

20. E.H. Mamdani, S. Assilian, An experiment in linguistic synthesis with a fuzzy logic controller, Int. J. Man-Machine Studies 7, 1-13 (1975) 